\title{
Searching for a Dark Photon Signal with PADME
}

\author{
F. Oliva on behalf of the PADME collaboration \\ Università del Salento and INFN Lecce, Via per Arnesano, 73100 Lecce, Italy
}

\begin{abstract}
PADME (Positron Annihilation into Dark Matter Experiment) is a fixed target experiment located at the Beam Test Facility (BTF) at the Laboratori Nazionali di Frascati (LNF) that searches for a massive dark photon $A^{\prime}$ in the process $e^{+} e^{-} \rightarrow \gamma A^{\prime}$, using a positron beam of energy up to $550 \mathrm{MeV}$. The experiment uses the missing mass technique, which allows to search for the dark photon in a model independent way. A sensitivity on the mixing constant $\epsilon>10^{-3}$ for a dark photon mass in the range $1 \leq \mathrm{m}_{\mathrm{A}^{\prime}} \leq 23.7 \mathrm{MeV} / \mathrm{c}^{2}$ can be achieved by collecting an integrated luminosity of $4 \times 10^{13}$ positrons on target.
\end{abstract}

Keywords: dark matter, dark photon, fixed target experiment

DOI: 10.31526/ ACP.BSM-2021.1

\section{DARK MATTER AND DARK PHOTON}

The Universe consists of ordinary baryonic matter for $5 \%$, dark matter for $26 \%$, and dark energy for the remaining $69 \%$, in according to the Standard Model of Cosmology.

The Standard Model of particle physics (SM) is not sufficient to explain the existence of the dark matter and nowadays models of Physics Beyond the SM (BSM) which foresee dark matter candidates are attracting the attention of the whole scientific community. A possible scenario is the introduction of a Dark Sector (DS) [1] in which dark particles are confined, that can only feebly interact with the world that we know through a portal.

One of the simplest models of the dark sector introduces an additional gauge symmetry $U^{\prime}(1)$ to describe the interactions among the dark particles. The hypothetical mediator between the SM and the DS could be the dark photon, the gauge boson of the dark matter. The simplest mechanism that could determine weak couplings between SM particles and the $A^{\prime}$ field is the mixing with the standard model photon; a kinetic mixing term can be then added in the Lagrangian:

$$
\mathcal{L}_{\text {mix }}=-\frac{\epsilon}{2} F_{\mu \nu}^{Q E D} F_{\text {dark }}^{\mu \nu}
$$

The lack of observations so far could be justified considering a small value of the mixing constant $\epsilon$. A new sector with new interactions could represent a possible solution to explain some of the known unsolved discrepancies between experiments and SM predictions, like the anomalous magnetic moment of the muon or the proton charge radius.

The dark photon can be either massless or massive; the latter case will be considered.

The mass of the dark photon could be generated through the Stueckelberg mechanism, which does not involve the spontaneous symmetry breaking, or can be acquired via a Higgs-like mechanism, through the SM Higgs or a new dark Higgs.

\subsection{Dark photon search}

Experiments at accelerators can probe the parameter space $\left(m_{A^{\prime}}, \epsilon\right)$ and set some constraints for $m_{A^{\prime}}>1 \mathrm{MeV}$.

The phenomenology of the dark photon affects the search techniques. Two decay modes have to be considered for the dark photon, depending on the comparison between the dark photon mass and the mass of the hypothetical dark particle $\chi$ of the dark sector:

- visible decays: $A^{\prime} \rightarrow S M+S M$ if $2 m_{e}<m_{A^{\prime}}<2 m_{\chi}$.

The decay in DS particles is kinematically forbidden for a mass of the dark photon two times the mass of any dark particle and the $A^{\prime}$ can only decay into SM particles if $m_{A^{\prime}}>2 m_{e}$. A branching ratio of $100 \%$ into $e^{+} e^{-}$is foreseen for a mass of a dark photon with mass lower than $20 \mathrm{MeV}$.

The width can be calculated as follows:

$$
\Gamma_{A^{\prime} \rightarrow f \bar{f}}=\frac{1}{3} \alpha \epsilon^{2} m_{A^{\prime}} \sqrt{1-\frac{4 m_{f}^{2}}{m_{A^{\prime}}^{2}}}\left(1+\frac{2 m_{f}^{2}}{m_{A^{\prime}}^{2}}\right)
$$

where $m_{f}$ is the mass of the SM fermion and $\alpha$ is the fine structure constant.

For a very low mixing constant $\epsilon$ the dark photon would be long lived, as the decay time is proportional to $1 /\left(\alpha \epsilon^{2} m_{A^{\prime}}\right)$. In the particular case of $m_{A^{\prime}}<2 m_{\chi}$ with $m_{A^{\prime}}<m_{e}$, the only accessible process is the production of $3 \gamma \mathrm{s}$ in the final state, via electron box diagram. Even in this case the $A^{\prime}$ is long lived; 

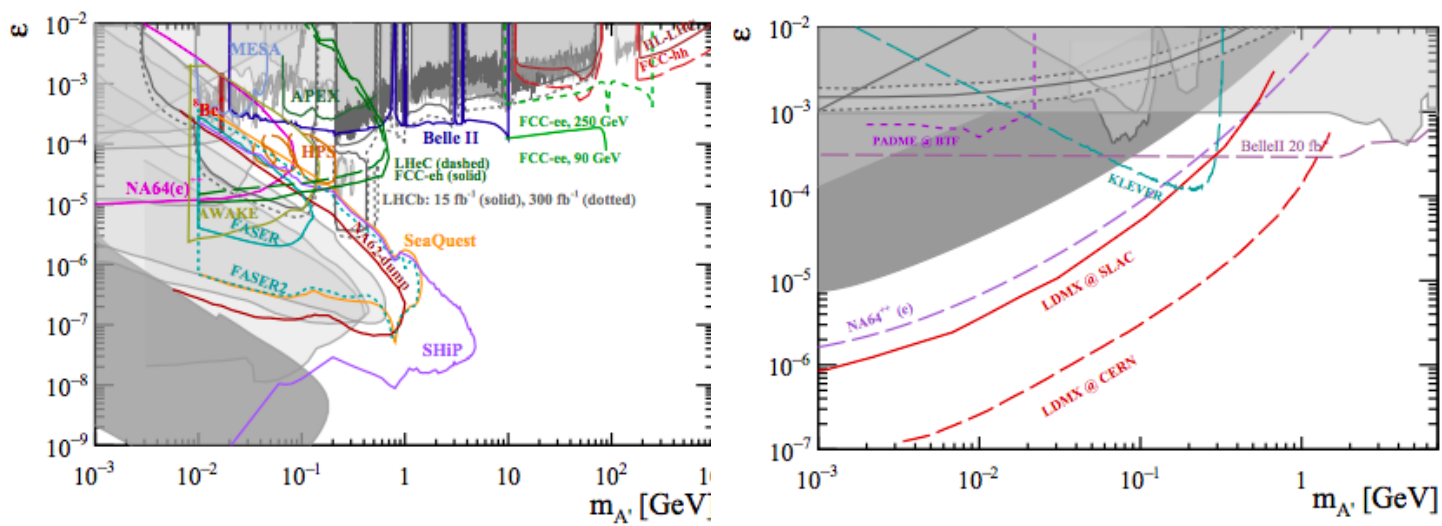

FIGURE 1: Regions of the parameter space already probed (in grey) or to be probed by future experiments (coloured lines) in the hypothesis of visible (left) or invisible (right) decay of the dark photon.

- invisible decays: $A^{\prime} \rightarrow \chi \chi$, if $m_{A^{\prime}}>2 m_{\chi}$.

The decay width is:

$$
\Gamma_{A^{\prime} \rightarrow \chi \bar{\chi}}=\frac{1}{3} \alpha_{D^{\prime}} m_{A^{\prime}} \sqrt{1-\frac{4 m_{\chi}^{2}}{m_{A^{\prime}}^{2}}}\left(1+\frac{2 m_{\chi}^{2}}{m_{A^{\prime}}^{2}}\right)
$$

where $\alpha_{D}$ represents the coupling of the dark photon to the dark matter.

A reasonable assumption is $\alpha_{D}>>\epsilon^{2}$, implying that the invisible decay dominates. The decay time in this case strictly depends on the $\alpha_{D}$ value $\left(\tau \propto 1 /\left(\alpha_{D} m_{A^{\prime}}\right)\right)$.

Many experiments around the world are searching for a dark photon, assuming visible or invisible decay mode, setting some constraints in the parameter space $\left(m_{A^{\prime}}, \epsilon\right)$. Regarding the visible decays (Fig.1, left), most of the regions are excluded by different experiments. Instead, some regions of the parameter space with the dark photon which decays into invisible states (Fig.1, right) are still open.

PADME is the first experiment designed and built to search for a dark photon in a model-independent way.

\section{THE PADME EXPERIMENT}

PADME (Positron Annihilation into Dark Matter Experiment) [2] is a fixed target experiment located at the Beam Test Facility (BTF) [3] at the Laboratori Nazionali di Frascati (LNF) that searches for a massive dark photon $A^{\prime}$ in the process $e^{+} e^{-} \rightarrow \gamma A^{\prime}$, using a positron beam of energy up to $550 \mathrm{MeV}$.

PADME exploits the missing mass technique and the dark photon mass can be computed considering the conservation of the quadri-momentum:

$$
m_{\text {miss }}^{2}=\left(P_{e^{+}}+P_{e^{-}}-P_{\gamma}\right)^{2},
$$

where $P_{e^{+}}, P_{e^{-}}$and $P_{\gamma}$ are the 4-momenta respectively of the positron beam, the target electron and the emitted photon.

The hypothetical dark photon event has a peak at the dark photon mass $\mathrm{m}_{A^{\prime}}$ over a smooth background.

The PADME beam is characterised by a bunch of about 25000 positrons, with a bunch length up to $300 \mathrm{~ns}$ and rate $50 \mathrm{~Hz}$. The maximum energy of the positron bunch delivered in PADME is $550 \mathrm{MeV}$, hence the maximum dark photon mass probed is equal to $23.7 \mathrm{MeV} / \mathrm{c}^{2}$.

\section{THE PADME DETECTOR}

The PADME detector (shown in Fig. 2) is composed of a thin diamond target, a magnet, which bends the beam outside the experimental acceptance, a high resolution electromagnetic calorimeter (ECAL), a fast small angle electromagnetic calorimeter (SAC) and a charged particle veto system (VETO).

Two beam monitors were also installed: MIMOSA, a monolitic Silicon detector, in the interaction region, and TIMEPIX, a hybrid Silicon pixel detector, in the beam dump region. A vacuum vessel hosts the diamond target and the charged particle veto system.

\subsection{Active diamond target}

The PADME target should meet three important requirements: 


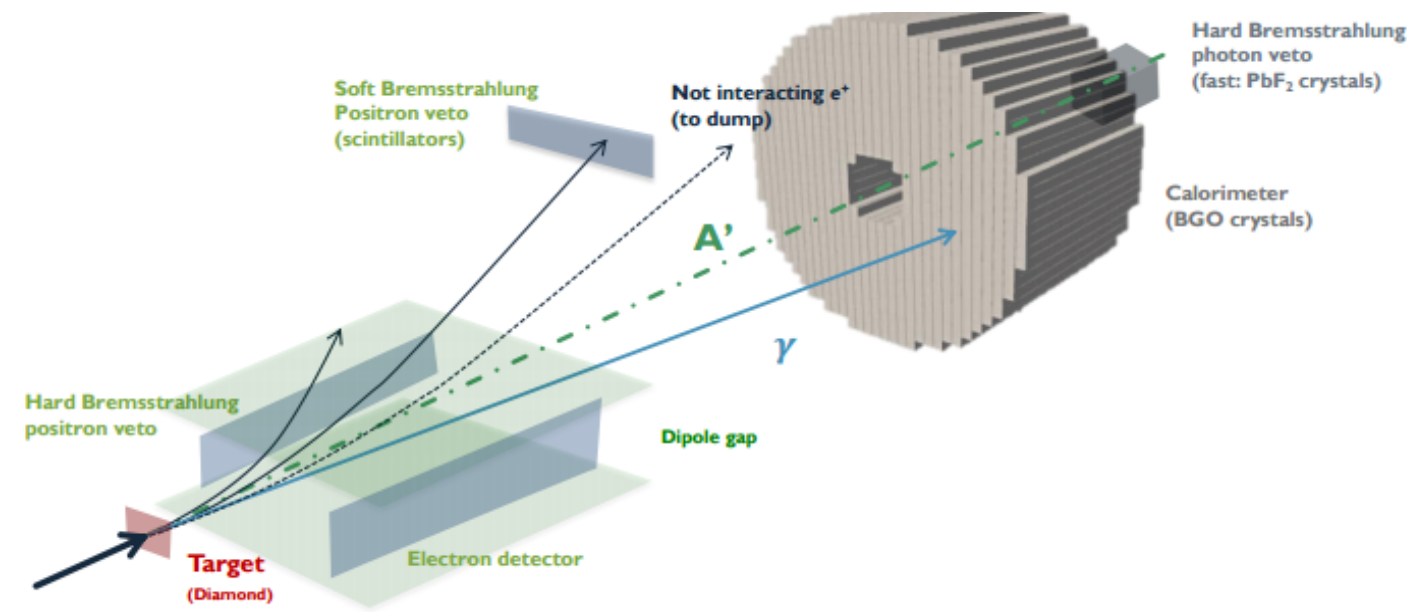

FIGURE 2: Layout of the PADME detector.
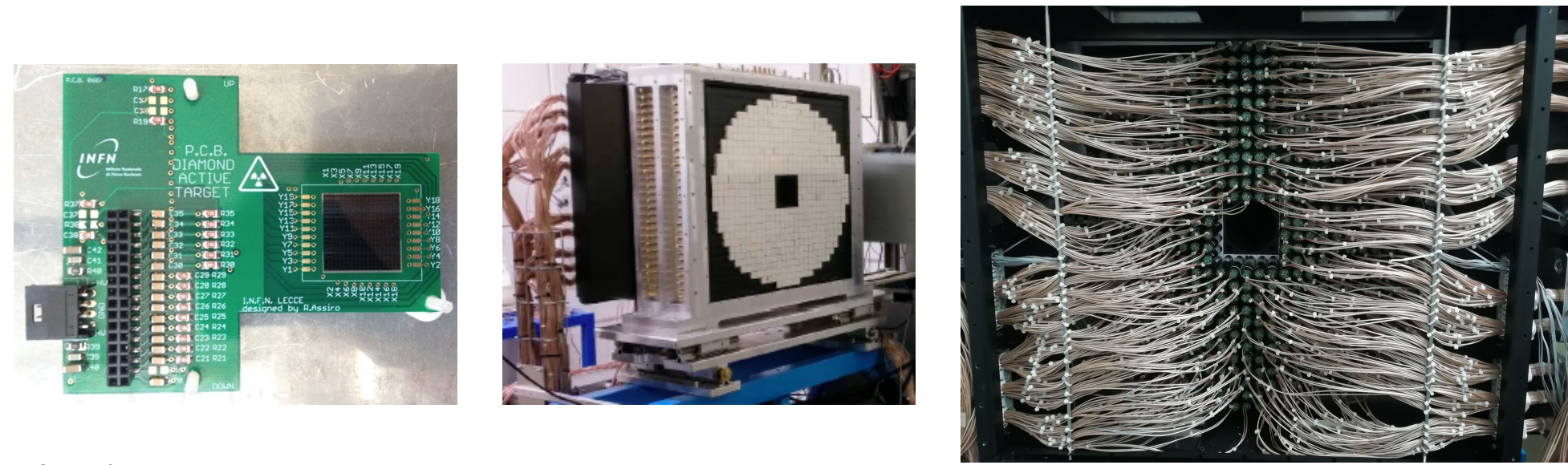

FIGURE 3: On the left the diamond target of the experiment assembled to the inner board; the front side and back side of ECAL are respectively shown in the center and on the right.

- its material must have a low atomic number to reduce the rate of Bremsstrahlung interactions $\left(\propto Z^{2}\right)$, main background of the experiment. For this reason, the diamond $(Z=6)$ was chosen as suitable material for the active target of the experiment;

- it must be active, providing the $\mathrm{X}$ and $\mathrm{Y}$ beam profiles in order to reconstruct the interaction point. This is related to the possibility to improve the missing mass resolution if the $A^{\prime}$ production point on the target is measured with a precision lower than $1 \mathrm{~mm}$;

- it has to provide the particle multiplicity for each bunch, namely the instantaneous luminosity of the experiment.

The active diamond target of PADME is a double sided strip detector, $100 \mu \mathrm{m}$ thick and $2 \times 2 \mathrm{~cm}^{2}$ area. The $19 \times 19$ strips on the two sides are oriented along orthogonal directions and perpendicularly to the beam, with a pitch of $1 \mathrm{~mm}$. This is an innovative "full carbon" detector, with the graphite strips realized by means of an excimer laser ArF ( $\lambda=193 \mathrm{~nm}, \tau=20 \mathrm{~ns})$ in the L3 Laboratory of Lecce.

The diamond was mechanically connected to the inner board by spots of Araldite glue, and electrically connected on the back side by filling special holes of the inner boards with a conductive glue and on the front by means of wire bondings. The diamond assembled on the inner board is shown in Fig. 3, left. The front-end electronics chosen is made of two evaluation boards of the AMADEUS chip, electrically connected to the inner board by two rigid connectors on both sides to readout $16 \mathrm{X}$ and $16 \mathrm{Y}$ strips [4].

\section{2. $E C A L$}

The electromagnetic calorimeter (ECAL) must detect the photon hypothetically produced together with the dark photon and measure its energy with high precision.

It is made of 616 BGO crystals of $2.1 \times 2.1 \times 23.0 \mathrm{~cm}^{3}$ size each, arranged in a cylindrically shaped array of $29 \mathrm{~cm}$ radius at a distance of $\sim 3.4 \mathrm{~m}$ from the target. In addition, a squared hole in the center, of dimension of $5 \times 5$ crystals, was made to prevent the detection of a high rate of Bremsstrahlung photons, mostly emitted in the forward direction. Each BGO crystal was coupled to 
a photo-multiplier HZC XP1911 type B, glued with ELJEN EJ-500 optical cement and covered with three layers of a white painting ELJEN EJ-510 [5]. A picture of ECAL is shown in Fig. 3 (front side in the center and back side on the right).

\subsection{Charged particle veto system}

The Bremsstrahlung events can be rejected thanks to a charged veto system, by detecting the positron emitted in coincidence with the photon in the calorimeters. The positron emitted in coincidence could be detected both by the Positron Veto (PVeto) inside the PADME magnet, which covers the internal vertical wall of the vacuum chamber, and by the High Energy Positron (HEP) veto, which covers a small region located near the beam dump. They are respectively made of an array of 90 and 16 scintillating bars. An additional Electron Veto (EVeto), made of 96 scintillating bars, is located facing the PVeto, in the magnet. The three arrays of bars are rotated around their longitudinal axis by $0.1 \mathrm{rad}$ to minimize geometrical inefficiencies and are held together thanks to an Aluminium support. Each scintillating bar of size $10 \times 10 \times 178 \mathrm{~mm}^{3}$ is made of a plastic polystyrene-based material with $1.5 \%$ concentration of POPOP and they are produced by UNIPLAST. In addition, a groove in the center of each bar hosts a wavelength shifter (WLS) fiber BCF-92, glued with Eljen EJ500 optical epoxy cement. Finally, silicon photo-multipliers (SiPMs) Hamamatsu 13360 allow the conversion of the photon into signals [6].

\subsection{Small Angle Calorimeter}

The small angle calorimeter is a matrix of $5 \times 5 \mathrm{PbF}_{2}$ crystals of size $3 \times 3 \times 14 \mathrm{~cm}^{3}$, coupled to R13478UVPMT Hamamatsu photomultipliers. This detector is located just behind the ECAL, in order to totally cover the geometrical acceptance of the hole, being capable to sustain a high rate of photons [7].
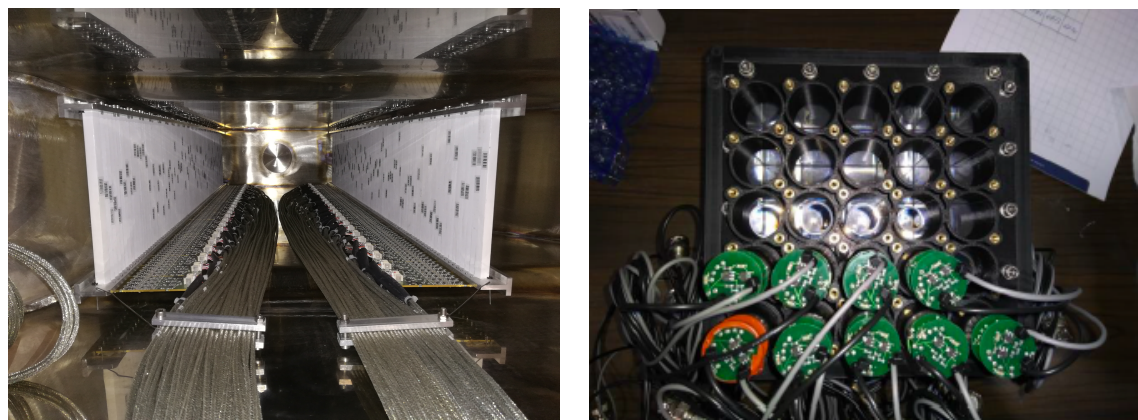

FIGURE 4: On the left the vacuum vessel which hosts the PVeto on the right and the EVeto on the left. On the right the SAC during the assembly.

\section{DARK PHOTON SIGNAL WITH PADME}

\subsection{PADME signature}

The efficiency on the signal selection is related to the knowledge of the $A^{\prime}$ production point on the target, together with a good measurement of the photon energy and direction.

A candidate dark photon event should meet the following requirements:

- only one photon in ECAL inside a radial fiducial region with no other photons in ECAL and in SAC within \pm 2 ns, to reject the annihilation final state in two or three photons;

- no positrons in the positron veto in \pm 2 ns coincidence with the photon detected by ECAL and with energy summed to the photon energy compatible with the beam energy, to reject events of Bremsstrahlung.

\subsection{PADME background}

A good background rejection is a fundamental requirement of the PADME experiment. The background processes are in particular Bremsstrahlung, annihilation in two or three photons and Bhabha scattering.

The most dangerous background is the Bremsstrahlung. A positron of the beam which interacts with the a nucleus of the target can be slowed down, emitting a photon:

$$
e^{+} N \rightarrow e^{+} N \gamma
$$

The Bremsstrahlung background can be suppressed requiring the time coincidence between the photon detected by ECAL and the positron passing through the veto bars (Fig. 5, a), with a total energy equal to the beam energy. Sometimes, the positron could escape from the veto acceptance; in this case the photon detected by ECAL represents an an irreducible background (Fig. 5 , b).

Another source of background is the SM annihilation in 2 or $3 \gamma \mathrm{s}$ :

$$
e^{+} e^{-} \rightarrow \gamma \gamma(\gamma)
$$



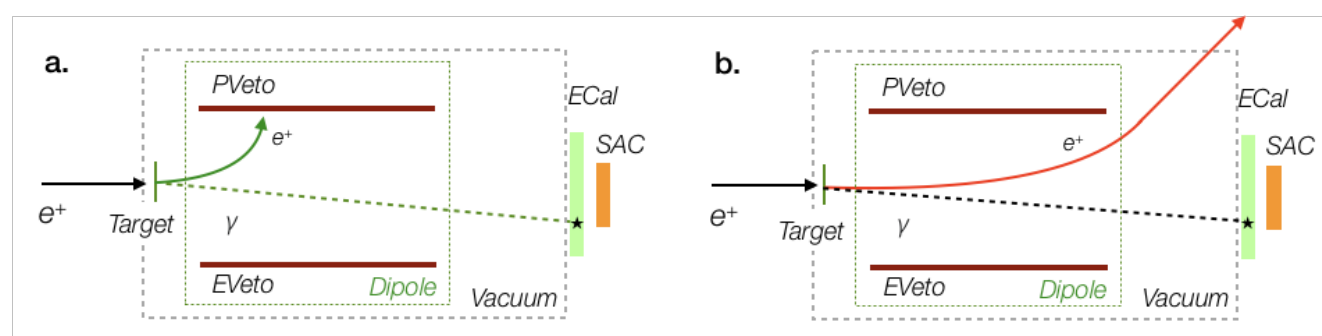

FIGURE 5: Bremsstrahlung process. On the left an example of background suppression, on the right the irreducible background to the dark photon signal.
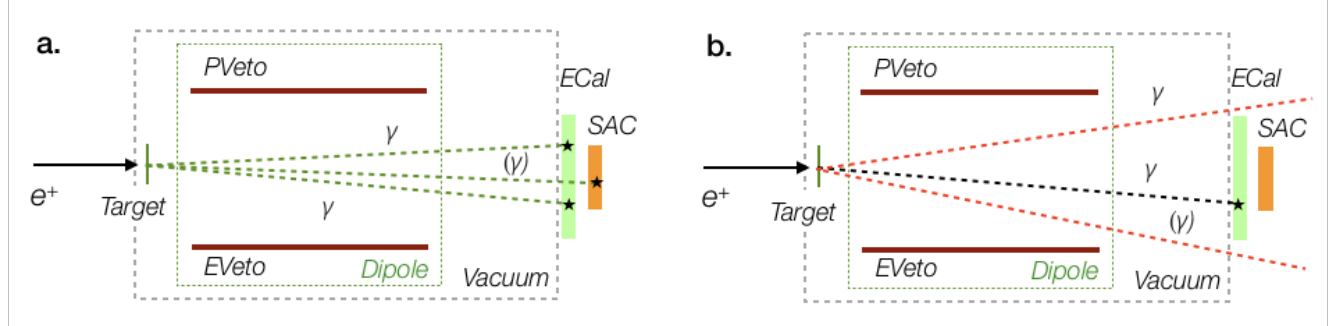

FIGURE 6: Annihilation (in 2 or $3 \gamma$ s) process. On the left an example of background suppression, on the right the irreducible background to the dark photon signal.

The first can be suppressed requiring two ECAL photons in time coincidence, symmetric in the azimuthal angle and with the sum of their energies equal to the energy of the beam. An important vetoing role has the SAC, which allows to reject the $3 \gamma \mathrm{s}$ final state, detecting the forward photon emitted in coincidence with the other two (Fig. 6, a). The irreducible background in $3 \gamma \mathrm{s}$ case is represented by a single photon detected by ECAL, with the second and third escaping not only ECAL, but also SAC (Fig. 6 , b).

\subsection{PADME sensitivity}

The PADME sensitivity depends by event in-bunch pile-up and beam background. The upper limit of the dark photon mass is 23.7 MeV $/ \mathrm{c}^{2}$, depending on the maximum energy reachable for positrons with the existing LINAC. The maximum number of positrons per bunch sets the lower limit on the mixing constant $\epsilon$ in PADME of about $10^{-3}$. The recent beam-line upgrades allowed to raise the delivered number of positrons per bunch up to 30000, keeping the pile-up at a manageable level for the detectors, extending the bunch duration up to $300 \mathrm{~ns}$. In this way, the time to reach the project integrated luminosity can be shortened: in 6 month a data sample of $1 \times 10^{13}$ POT can be collected while in 2 years a sample of $4 \times 10^{13}$ POT can be reached. In Fig. 7 is reported the ideal excluded region of the parameter space $\left(\mathrm{m}_{A^{\prime}}, \epsilon^{2}\right)$ for these two different values of the integrated luminosity. The region of the dark photon mass in the range $10-100 \mathrm{MeV}$ and $\epsilon \sim 10^{-3}$ is of particular interest as it could account for the discrepancy between the measured and the theoretical value of the anomalous magnetic momentum of the muon [8].

\section{PADME DATA TAKING}

The PADME data taking periods strictly depend on the beam features. Two kind of positron beams can be exploited by PADME, depending on the way they are originated from the electrons:

- primary positron beam with positrons directly produced in the LINAC thanks to a W-Re positron converter placed on the experimental beam line just after the first accelerating sections of the electrons, far from the experimental hall;

- secondary positron beam with positrons produced in the interactions of the electron beam in a Cu target placed just before the entrance of the experimental hall.

The detector was fully installed in September 2018 and two data taking periods were taking place:

- Run 1 from October 2018 to March 2019 and an additional data period in July 2019, with an old beam line, with a Be window separator between the LINAC and PADME vacuum. During the first part of this run a secondary beam was used while in the last part a primary beam was exploited.

- Run 2 from September 2020 to December 2020, with an additional data taking period in July 2020, with a new beam line, with a Mylar window separator between the LINAC and PADME vacuum moved far from the PADME detector. This choice helped to reduce the beam background. During this run only a primary beam was exploited. Run 2 allowed to collect a total of $5 \times 10^{12}$ positrons on target. 


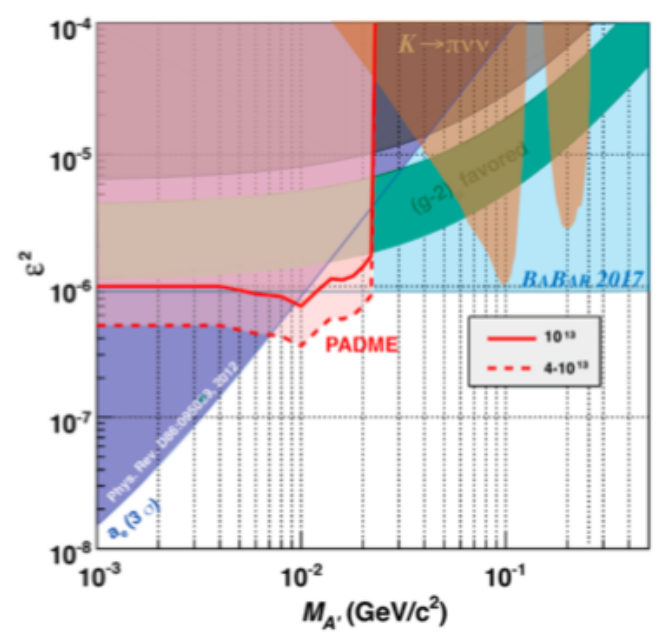

FIGURE 7: PADME exclusion region of the parameter space for a project luminosity of $10^{13}$ or $4 \times 10^{13}$ positrons on target, which can be reached in 6 months and 2 years, respectively, with the recent beam-line upgrades [9].

A reliable Data Acquisition system (DAQ) and Detector Control System (DCS), together with a detailed on-line monitoring, were essential tools for the data taking.

shift organisation

\section{ADDITIONAL SEARCHES WITH PADME}

The missing mass technique exploited by the PADME experiment allows to test new physics models beyond the dark photon, searching for any other light dark particle $X$. The final state could be written as follows:

$$
e^{+} e^{-} \rightarrow \gamma X
$$

Some studies are ongoing to investigate with the PADME experimental set-up the existence of an Axion-Like-Particle or a Dark Higgs.

A new approach must be exploited to search for the X17 [10] boson with PADME, which requires some changes in the experimental set-up [11].

\subsection{Axion-like-particles}

A possible pseudo-scalar spin-0 mediator between the SM and the DS could be an Axion-Like Particle (ALP). A visible decay into a $e^{+} e^{-}$pair or $2 \gamma$ is foreseen if no other dark sector particle lighter than the ALP, $\mathrm{m}_{A L P} \mathrm{im}_{\chi}$, exists. ALPs couple to bosons (like photons with coupling $\mathrm{g}_{a \gamma \gamma}$ ) and fermions (like $\mathrm{e}^{-}$with coupling $\mathrm{g}_{a e e}$ ) and, in general, without relations between mass and coupling (unlike for QCD axions). The PADME experiment can search for ALPs decaying in visible or invisible states [12]. If an ALP decays into visible states such as $e^{+} e^{-}$or two photons, the accessible finale state for PADME would be an $e^{+} e^{-}$pair in time with a photon or $3 \gamma \mathrm{s}$ in time coincidence. If an ALP decays into invisible states, the accessible finale state for PADME would be the same as the dark photon one: a single SM photon in ECAL and missing mass. For masses lower than $100 \mathrm{MeV}$ the ALP could be long lived and the PADME signature would be the same of the invisible case. According to some estimations, a thousand ALP events for a mass of $22 \mathrm{MeV}$ and $\mathrm{g}_{a e e}=1$ would be produced in 2 years of data taking with $60 \%$ of efficiency and $28000 \mathrm{e}^{+} / \mathrm{bunch}$ (for a total of about $4 \times 10^{13} \mathrm{NPOT}$ ), totally independent from the $\mathrm{g}_{a \gamma \gamma}$ value [13]. Limits on free parameters for invisible channel were set following this study.

\subsection{Dark Higgs}

Under the hypothesis of the existence of a dark Higgs, the dark photon could acquire mass through a Higgs-like mechanism. Supposing a similar mass for the dark photon and the dark Higgs, the process $e^{+} e^{-} \rightarrow A^{\prime} h^{\prime}$ can be searched for. A dark-Higgs could decay into two dark photons if $m_{h^{\prime}}>2 m_{A^{\prime}}$; in this way the final state of the $e^{+} e^{-}$annihilation would be represented by 6 leptons:

$$
e^{+} e^{-} \rightarrow A^{\prime} h^{\prime} \rightarrow A^{\prime} A^{\prime} A^{\prime} \rightarrow 3\left(e^{+} e^{-}\right)
$$

PADME could search this particular final state requiring the coincidence of six charged particles exploiting both the PVeto and the EVeto [14]. In case of a invisible decay of the dark Higgs or a long-lived one, the accessible final state would be a $e^{+} e^{-}$pair from the $A^{\prime}$ decay and a missing mass component. 


\subsection{Protophobic boson search at PADME}

An Hungarian group from Atomki (Debrecem) recently built a spectrometer with the aim of performing a simultaneous energy and angular correlation measurement of electron-positron pairs in the decay of excited ${ }^{8}$ Be nuclei [15]. An excess of pair production with a well defined separation angle was measured, that can be justified by the production of a new particle named X17. In addition, another anomaly in ${ }^{4} \mathrm{He}$ nuclear transitions was reported by the same group [16]. A protophobic X boson could be a possible explanation of these observed phenomena [17].

In particular, a solution could be the existence of a dark photon with mass $m_{A^{\prime}} \sim 17 \mathrm{MeV}$. The resonant production mechanism $e^{+} e^{-} \rightarrow A^{\prime} \rightarrow e^{+} e^{-}$is a favoured production mode, being of the order of $\epsilon^{2} \alpha$. The decay length of the $A^{\prime}$ so produced does not depend on the boson mass $\left(\ell_{\epsilon} \sim 3 / 2 m_{e} \alpha \epsilon^{2}\right)$ for a given value of $\epsilon$. This allows to probe the range of mass up to the kinematic limit, with the same sensitivity.

PADME could search for this dark photon with a few proper experimental set-up changes [11]. One of the requirement is the reduction of the beam energy, to about $282 \mathrm{MeV}$ to investigate the resonance production. In addition, the probability of resonant production would increase using a thick target of a material with large atomic number, for example Tungsten (W). With such a thick target, the positrons would lose energy inside it before annihilating producing a sample of positrons of almost continuously varying energy. This would solve the issue of setting the beam to the exact center of mass energy corresponding to the narrow resonance. The positrons with the correct energy would then annihilate with atomic electrons via a resonant s-channel exchange of the $A^{\prime}$. If the $A^{\prime}$ travels through all the target, decaying next to the exit, the $\mathrm{e}^{+} \mathrm{e}^{-}$pair of the final state would be detected by the charged particle detectors. The thick target would also act as a hermetic dump for the other positrons of the beam, reducing the background from SM processes. The excess of events at a value of the invariant mass corresponding to the dark photon mass would be identified by the measurement of the momentum of the positron and electron. A hypothetical luminosity of about $10^{18}$ $\mathrm{POT} /$ year, should be also foreseen, compatible with the possibility to stand the resulting pile-up.

\section{CONCLUSIONS}

PADME is a small scale experiment designed and built to search for a dark photon signal using the missing mass technique exploiting a bunched positron beam. The detector was fully installed in September 2018; the data taking started in October 2018 and ended in December 2020, with relatively long shutdowns of several months. Thanks to a good shift operation, a reliable DAQ, DCS and on-line monitoring, the Run 2 from September to December 2020 allowed to reach an integrated luminosity of $5 \times 10^{12}$ positrons on target with low background conditions. The analysis of the data taken is currently ongoing. The technique used by PADME is model independent, allowing the search for other hypothetical particles like the Axion-Like-Particle or the Dark Higgs. A possible opportunity for PADME in the next future could be the X17 boson search, with some changes in the set-up.

\section{References}

[1] J. Alexander et. al., "Dark Sectors 2016 Workshop: Community Report”, (2016) arXiv:1608.08632 [hep-ph];

[2] M. Raggi and V. Kozhuharov, "Proposal to search for a dark photon in positron on target collisions at DA $\Phi$ NE linac", Adv. High Energy Phys. 509, $959802(2014)$;

[3] A.Chigo et al., "Commissioning of the DAFNE beam test facility", Nucl. Instrum. Meth. A 515 (2003), pp. 524-542;

[4] F. Oliva on behalf the PADME collaboration, "Searching for a dark photon with PADME at LNF: status of the active diamond target', Nucl. Instrum. Meth. A 936 (2019), 697-698 doi:10.1016/j.nima.2018.10.135

[5] P. Albicocco et al., "Characterisation and performance of the PADME electromagnetic calorimeter", JINST 15 (2020) n.10, T10003 doi:10.1088/1748-0221/15/10/T10003 [arXiv:2007.14240 [physics.ins-det]];

[6] F. Oliva on behalf the PADME collaboration, "The charged particle veto system of the PADME experiment", Nucl. Instrum. Meth. A 936 (2019), 259-260 doi:10.1016/j.nima.2018.10.147

[7] A. Frankenthal et al., "Characterization and performance of PADME's Cherenkov-based small-angle calorimeter", Nucl. Instrum. Meth. A 919 (2019), 89-97 doi:10.1016/j.nima.2018.12.035 [arXiv:1809.10840 [physics.ins-det]];

[8] P. A. Zyla et al. [Particle Data Group], “Review of Particle Physics”, PTEP 2020 (2020) n.8, 083C01 doi:10.1093/ptep/ptaa104;

[9] M. Raggi on behalf the PADME collaboration, "The PADME experiment", Frascati Phys. Ser. 66 (2018), 337-344;

[10] A. J. Krasznahorkay et al., "Observation of Anomalous Internal Pair Creation in Be8 : A Possible Indication of a Light, Neutral Boson", Phys. Rev. Lett. 116 (2016) n.4, 042501 doi:10.1103/PhysRevLett.116.042501 [arXiv:1504.01527 [nucl-ex]];

[11] E. Nardi et al., "Resonant production of dark photons in positron beam dump experiments", Phys. Rev. D 97 (2018) n.9, 095004 doi:10.1103/PhysRevD.97.095004 [arXiv:1802.04756 [hep-ph]];

[12] F. Giacchino, "A light dark matter portal: The axion-like particle", Frascati Phys. Ser. 69 (2019), 206-211;

[13] L. Darmé, F. Giacchino, E. Nardi and M. Raggi, "Invisible decays of axion-like particles: constraints and prospects", [arXiv:2012.07894 [hepph]];

[14] G. Martelli, P. Ciafaloni and M. Raggi, "Searching for New Physics with multilepton events at PADME", [arXiv:2102.13374 [hep-ph]];

[15] J. Guly et al., "A pair spectrometer for measuring multipolarities of energetic nuclear transitions", Nucl. Instrum. Meth. A 808 (2016), 21-28 doi:10.1016/j.nima.2015.11.009 [arXiv:1504.00489 [nucl-ex]];

[16] A. J. Krasznahorkay et al., "New evidence supporting the existence of the hypothetic X17 particle", [arXiv:1910.10459 [nucl-ex]];

[17] J. L. Feng et al. "Particle physics models for the $17 \mathrm{MeV}$ anomaly in beryllium nuclear decays", Phys. Rev. D 95 (2017) n.3, 035017 doi:10.1103/PhysRevD.95.035017 [arXiv:1608.03591 [hep-ph]]. 